\title{
LIQUENS UTILIZADOS COMO BIOMONITORES DA QUALIDADE DO AR NO PARQUE DA JAQUEIRA - RECIFE - PERNAMBUCO
}

\section{LICHENS USED AS BIOMONITORS OF AIR QUALITY IN THE JAQUEIRA PARK - RECIFE - PERNAMBUCO}

\author{
Andrezza Karla de Oliveira Silva \\ Mestranda do Programa de Pós-Graduação em Geografia \\ Universidade Federal de Pernambuco \\ andrezzakarla86@hotmail.com \\ Iwelton Madson Celestino Pereira \\ Mestrando do Programa de Pós-Graduação em Geografia \\ Universidade Federal de Pernambuco \\ madson85@hotmail.com \\ Nicácio Henrique da Silva \\ Professor Doutor do Depto. de Bioquímica \\ Universidade Federal de Pernambuco \\ nhsilva@uol.com.br \\ Fernando de Oliveira Mota-Filho \\ Professor Associado do Depto. De Ciências Geográficas \\ Universidade Federal de Pernambuco \\ fmf@elogica.com.br \\ Eugênia Cristina Gonçalves Pereira \\ Professora Titular do Depto. De Ciências Geográficas \\ Universidade Federal de Pernambuco \\ verticillaris@gmail.com
}

\begin{abstract}
RESUMO
O aumento da emissão de poluentes para atmosfera tem afetado a saúde humana, a biota e as oscilações climáticas. Desta forma, trabalhos que indiquem e/ou monitorem a qualidade do ar em áreas urbanas e rurais são importantes contribuições para a ciência. Organismos vivos são usados como indicadores ou monitores da qualidade do ar e, nesse contexto os liquens destacam-se por serem utilizados em diversas partes do mundo. Neste trabalho avaliou-sea qualidade do ar no parque da Jaqueira, Recife - PE a partir da flora liquênica existente. O parque foi dividido em 20 quadrantes de $60 \mathrm{~m}$ x 63 $\mathrm{m}$, onde se determinou o Índice de Pureza Atmosférica (IPA) e quantificados pigmentos fotossintéticosda espécie Parmotremapraesorediosum. Os resultados demonstraram que em todos os quadrantes foram encontradas espécies de liquens, no entanto $P$. praesorediosum só foi localizada em $55 \%$ do parque, área também considerada como
\end{abstract}


padrão de qualidade do ar de bom a superior. Os níveis baixo e intermediário da qualidade do ar foram detectados em locais onde predominam a construção civil e tráfego mais intenso de veículos, provocando a diminuição da diversidade liquênicapelospoluentes transportados pelos ventos através do parque.

Palavras-chave: poluição atmosférica; biomonitoramento; zoneamento da qualidade do ar; Recife; Parmotremapraesorediosum.

\begin{abstract}
The increasing of pollutants emission to the atmosphere has been affecting the human health, the biota and climate oscillations. This way, studies that indicate and/or monitoring the air quality in urban or rural areas are important contributions to the science. Living organisms are used as indicators or monitors of air quality, and in this context lichens stand out for their use in several parts of the world. In this research the air quality in Jaqueira park, Recife - PE, Brazil, was evaluated, from the existing lichen flora. The park was divided in 20 quadrants $(60 \mathrm{~m}$ x $63 \mathrm{~m})$, where the Index of Air Purity (IAP) was determined and quantified photosynthetic pigments of the lichen Parmotremapraesorediosum. The results showed that in all quadrants lichen species were registered, however the $P$. praesorediosum was only localized in $55 \%$ of the park, an areaalso considered with a good to superior air quality. The low and intermediary levels of air quality were detected where building and more intense traffic were predominant, causing a decreasing of lichen diversity due to the pollutants transported by wind throughout the park.
\end{abstract}

Keywords: atmospheric pollution; biomonitoring; air quality zoning; Recife; Parmotremapraesorediosum.

\title{
INTRODUÇÃO
}

A poluição atmosférica temse tornado um dos maiores desafios a serem enfrentados pela sociedade, devido aos diversos fatores de ordem natural, social e econômica. Os processos industriais e de geração de energia, os veículos automotores e as queimadas são, dentre as atividades antrópicas, as maiores causas da procedência de substâncias poluentes na atmosfera, a maioria delas tóxicas, causando danos à saúde humana efortes impactos à biota.

A contaminação do ar afeta o clima das áreas urbanas de diversas formas. O equilíbrio energético das cidades sofre interferências, pois os poluentes refletem, dispersam e absorvem a radiação solar. A poluição apresenta efeitos distintos sobre os seres vivos. Os vegetais, em particular, sofrem sobremaneira com a interferência direta dos poluentes sobre sua capacidade fotossintetizante, pois afetam o balanço energético 
das superfícies das folhas (AYOADE, 1998). Por outro lado, as árvores tornam-se ferramentas na filtragem dos poluentes, como também ajudam a amenizar os efeitos das ilhas de calor urbanas(NOWAK et al., 2000).

A qualidade do ar pode ser avaliada em diferentes escalas:local, regional, e global, através da mensuração das emissões, da utilização de modelos matemáticos e de medidas de concentrações ambientais dos poluentes usando métodos físico-químicos (KLUMPP et al., 2011). O uso de monitores biológicos para a detecção das mudanças ocorridas no meio ambiente é uma técnica rápida e eficaz, sendo o biomonitoramento da qualidade do ar a partir dos liquens um método sistemático que quantifica e qualifica respostas de organismos vivos à poluição.

Os liquens podem ser utilizados no biomonitoramento uma vez que são seres simbiônticos - uma associação de fungo e algaou cianobactéria - de ampla distribuição geográfica. Não possuem sistema radicular ou cutícula, o que facilita a retenção de nutrientes e poluentes dispersos no ar (material particulado, íons metálicos, elementos radioativos e gases tóxicos), que são absorvidos de forma higroscópica. Dessa maneira, são capazes de indicar a qualidade do ambiente e do ar atmosférico (NIEBOERet al., 1972; PILEGAARD, 1978; SEAWARD, 1977; MAZZITELLIet al., 2006)e, podem ser empregados como biomonitorespor sua sensibilidade às modificações ambientais e introdução de poluentes no ambiente, cujas respostas são obtidas a partir de mensurações fisiológicas e análises morfológicas e estruturais do talo (SEAWARD, 1993).

Diante daaglomeração das áreas urbanas, com a consequente intensificação do tráfego de veículos em espaços cada vez mais limitados, incremento da densidade populacional e, a falta de planejamento para ocupação das cidades, os impactos causados pelos poluentes atmosféricos são cada vez maispotencializados.

A qualidade do ar do município do Recife foi avaliada na década passada, sendo determinados pontos de alto, médio e baixo níveis de poluição, usando pigmentos fotossintéticos de plantas e liquens como parâmetros de mensuração (MOTA-FILHOet al., 2003). Liquens também foram avaliados como monitores de emissões de $\mathrm{SO}_{2} \mathrm{e} \mathrm{Pb}$ usado em área industrial no município de Belo Jardim (PE), sendo possível determinar o raio de ação do poluente e, identificar os fatores físico-ambientais capazes de dispersar e/ou barrar a dispersão dos poluentes (MOTA-FILHOet al., 2007). 
No município do Recife existem parques de diferentes dimensões, tendo destaque, dentre os de maior área e com equipamentos de lazer, o parque da Jaqueira. Este, apesar de considerado como umas das áreas mais verdes e conservadas do município está exposto a fontes de emissão de poluentes através de intensas vias de acesso que o circundam, dentre outras atividades antrópicas típicas de áreas urbanas.

Visto a eficácia dos liquens nos estudos de biomonitoramento e,a existência do zoneamento da qualidade do ar do município do Recife em uma primeira aproximação, neste trabalho objetivou-se trabalhar em escala de detalhe, realizando uma análise da influência de fontes poluidoras no entorno doparque da Jaqueira, usando liquens existentes na área como biomonitores.

Este tipo de estudo auxiliará na gestão de espaços verdes públicos, no intuito de preservar a densidade arbórea e a biota a ela associada, para melhoria da qualidade de vida das pessoas em zonas urbanas.

\section{MATERIAL E MÉTODOS}

O parque da Jaqueira (figura 1)está localizado no bairro de mesmo nome,zona norte da cidade do Recife, no Estado de Pernambuco. Inserido em uma das áreas nobres da cidade é circundado por uma densa urbanização - edificações e avenidas de intenso fluxo de veículos. O parque da Jaqueira teve sua inauguração em 1985e, é o maiorparque público da cidade do Recife com $70.000 \mathrm{~m}^{2}$ (PREFEITURA DO RECIFE, 2011). O parque é utilizado como área de lazer, possui pistas de cooper, bicicross, e áreas de diversão para crianças.

Figura 1 - Mapa de localização do parque da Jaqueira, Recife - PE. 

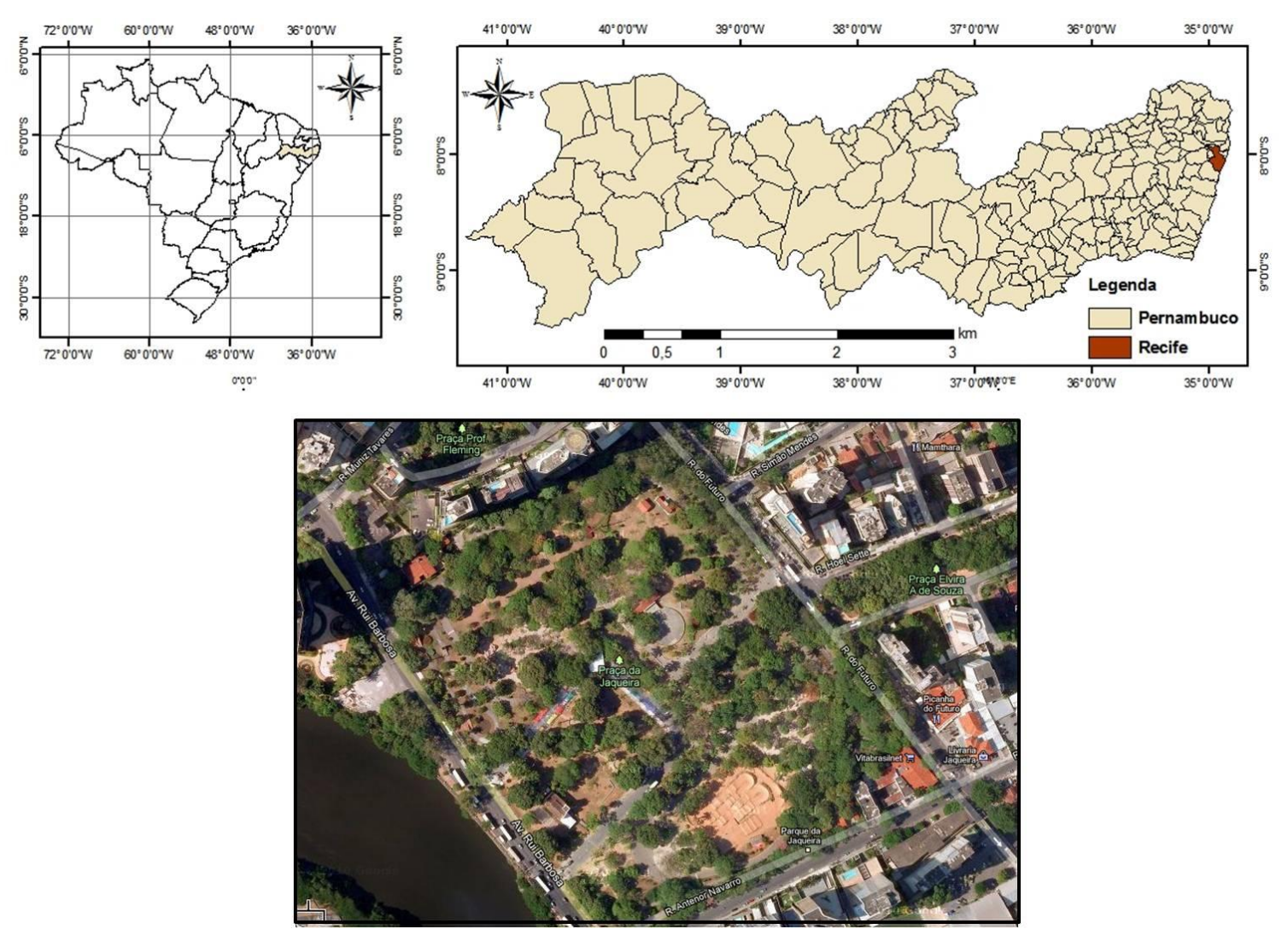

Fonte: IBGE, 2001. Licença ArcGIS 9.3 fornecida pelo SERGEO/UFPE. Google earth imagem parque da Jaqueira.

Para a realização do biomonitoramentoda qualidade do ar noparque da Jaqueira utilizaram-sedois métodos: a determinaçãodo Índice de Pureza Atmosférica (IPA) e a quantificação de pigmentos fotossintetizantes do líquen Parmotremapraesorediosum (Nyl.) Hale, espécie de ampla ocorrência na área e sensível à poluição (CÁCERES, 1996).

\section{Determinação do Índice de Pureza Atmosférica (IPA)}

Para aplicação do IPA no parque da Jaqueira, a área foi divida em 20 quadrantes de 60 × 63 m (figura 2), sendo selecionados seisforófitos(árvores que servem de substrato) com existência de liquens nas vertentes e no centro de cada quadrante.

Em cada forófito foi fixada uma malha com 25 quadrados, cada um com $5 \mathrm{~cm}$ de lado a 1,20 m de altura da base da árvore. Dentro da área da malha foram registrados os seguintes dados: o número de espécies liquênicas por forófito; frequência de espécies de liquens por quadrado da malha; e percentual de cobertura de liquens sob a superfície da 
malha.Os dados obtidos foram aplicados à fórmula desenvolvida por Cáceres (1996) adaptada de DeSloover\& Leblanc (1968).

Figura 2- Divisão do parque da Jaqueira em quadrantes para biomonitoramento da qualidade.do

ar.

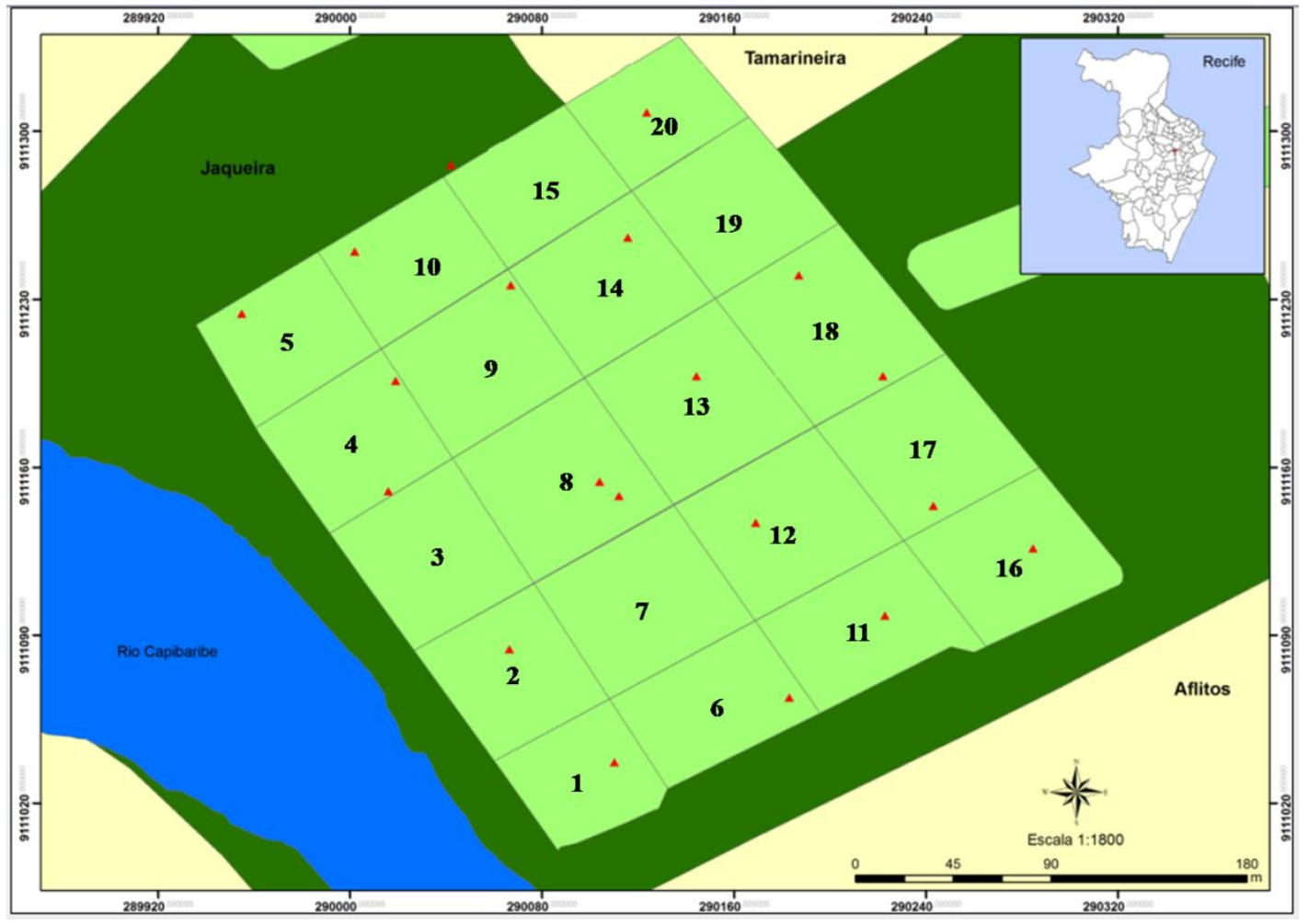

Fonte: IBGE, 2001. Licença ArcGIS 9.3 fornecida pelo SERGEO/UFPE.

\section{Análise de pigmentos fotossintetizantes}

Amostras deP. Praesorediosum foram coletadas para mensuração dos teores dos pigmentos fotossintetizantes clorofilas $\mathbf{a}$ e $\mathbf{b}$, e feofitinas $\mathbf{a}$ e $\mathbf{b}$. A extração procedeu-se a partir da infusão de $200 \mathrm{mg}$ de talo liquênico em $10 \mathrm{~mL}$ de acetona a 80\%, mantida no escuro por $48 \mathrm{~h}$, e posterior filtração. Os extratos foram lidos em espectrofotômetro BIOCHROM modelo Libra S 22 nos comprimentos de onda 666nm, 663nm, 645nm e $546 \mathrm{~nm}$. Os resultados das leituras foram aplicados em fórmulas para cálculo dos teores de clorofilas a e b (HILL, 1963) e feofitinas a e b (VERNON, 1960).

\section{Zoneamento da qualidade do ar no parque}

Para a realização do zoneamento da qualidade do ar no parque da Jaqueira, utilizaram-se os resultados obtidos no Índice de Pureza Atmosférica (IPA).Foi utilizada 
a variável visual cor e o método de implantação zonal para representação temática (MARTINELLI, 2003) dos dados do IPA. Estes dados foram plotados no mapa com a divisão do parque em 20 quadrantes dispostos em quatro cores diferentes correlacionadas com os níveis de degradação vermelho (baixo), amarelo (médio), azul (bom) e verde (muito bom).

\section{RESULTADOS E DISCUSSÃO}

Os resultados aqui obtidos possibilitam indicar a possível degradação ocorrente na área, fazendo uso de organismos já adaptados ao local. Mesmo com grande biomassa de liquens em alguns dos quadrantes do parque, havia o predomínio de algumas espécies, ou grande ocorrência de indivíduos crostosos (que se aderem ao substrato como se fizessem parte dele), que são mais resistentes aos impactos da poluição. Esses dados estão de acordo com Seaward (1977), que considera algumas espécies crosotsas, a exemplo Lecanoramuralis, considerada espécie urbana, mas também assim pode ser considerada a espécie foliosa (em forma de folha) Canoparmelia texana também resistente ao meio urbano (FUGA et al., 2008). Por isso, a presença de liquens em todos os quadrantes não sãotão bons resultados como se pode supor em primeira observação.

Nesse contexto, pode-se afirmar que a avaliação da poluição atmosférica a partir da micotaliquênica existente na área é um método que permite relacionar a presença ou ausência de espécies de liquens, sua diversidade, frequência, cobertura, danos externos e internos, com grau de poluição da área de estudo (LE BLANC et al., 1975).

Os dados de IPA foram divididos em quatro níveis levando-se em consideração os valores máximos e mínimos encontrados e, classificados como baixo, médio, bom e muito bom (Tabela 1). Verificou-se que 55\% do parque da Jaqueira estão enquadrados em níveis considerados como bom e muito bom com valores de IPA entre 25 a 38 . O zoneamento dos dados demonstra as áreas internas do parque com os maiores índices de IPA nos parâmetros entre bom (25\%) e muito bom (30\%) (figura 3). Estes dados tornaram-se mais evidentes pelo fato destas áreas serem mais arborizadas.

Yaakovet al. (1998) reforçam a importância das áreas verdes nos centros urbanos, devido à influência que a vegetação exerce nos parâmetros climáticos de diversas áreas e seus arredores, reduzindo a radiação, a temperatura, gerando a elevação da umidade e reduzindo a velocidade dos ventos. Em adição, a existência de uma 
densidade arbórea possibilita a formação de uma área protetora no entorno do parque, protegendo a biota no seu interior.

Em adição, Ribeiro et al. (1997) ao monitorarem o bosque do Museu Paraense Emílio Goeldi, em Belém (PA) verificaram que áreas mais protegidas pelas árvores tinham liquens menos afetados pela emissão de poluentes por veículos que transitavam nos seus arredores, ratificando os resultados encontrados neste trabalho.

Tabela 1. Classificação dos quadrantes do parque da Jaqueira, Recife - PE, segundo nível da qualidade do ar determinado pelos valores obtidos através do cálculo do índice de pureza atmosférica (IPA).

\begin{tabular}{|c|c|c|}
\hline Níveis/ valores de IPA & Quadrantes & IPA \\
\hline \multirow{4}{*}{$\begin{array}{c}\text { Baixo } \\
06 \text { a } 18\end{array}$} & 9 & 6,24 \\
\hline & 2 & 7,48 \\
\hline & 5 & 11,3 \\
\hline & 16 & 17,22 \\
\hline \multirow{5}{*}{$\begin{array}{l}\text { Médio } \\
18 \text { a } 25\end{array}$} & 4 & 18,15 \\
\hline & 15 & 19,76 \\
\hline & 10 & 20,7 \\
\hline & 13 & 21,46 \\
\hline & 3 & 22,62 \\
\hline \multirow{5}{*}{$\begin{array}{c}\text { Bom } \\
25 \text { a } 31\end{array}$} & 19 & 25,6 \\
\hline & 7 & 27,88 \\
\hline & 12 & 28,43 \\
\hline & 18 & 29,26 \\
\hline & 17 & 29,7 \\
\hline \multirow{5}{*}{$\begin{array}{c}\text { Muito bom } \\
\qquad 1 \text { a } 38\end{array}$} & 11 & 31,87 \\
\hline & 1 & 32,25 \\
\hline & 6 & 32,52 \\
\hline & 8 & 35,66 \\
\hline & 14 & 37 \\
\hline
\end{tabular}


Figura 3 - Zoneamento do parque da Jaqueira a partir dos dados do IPA.

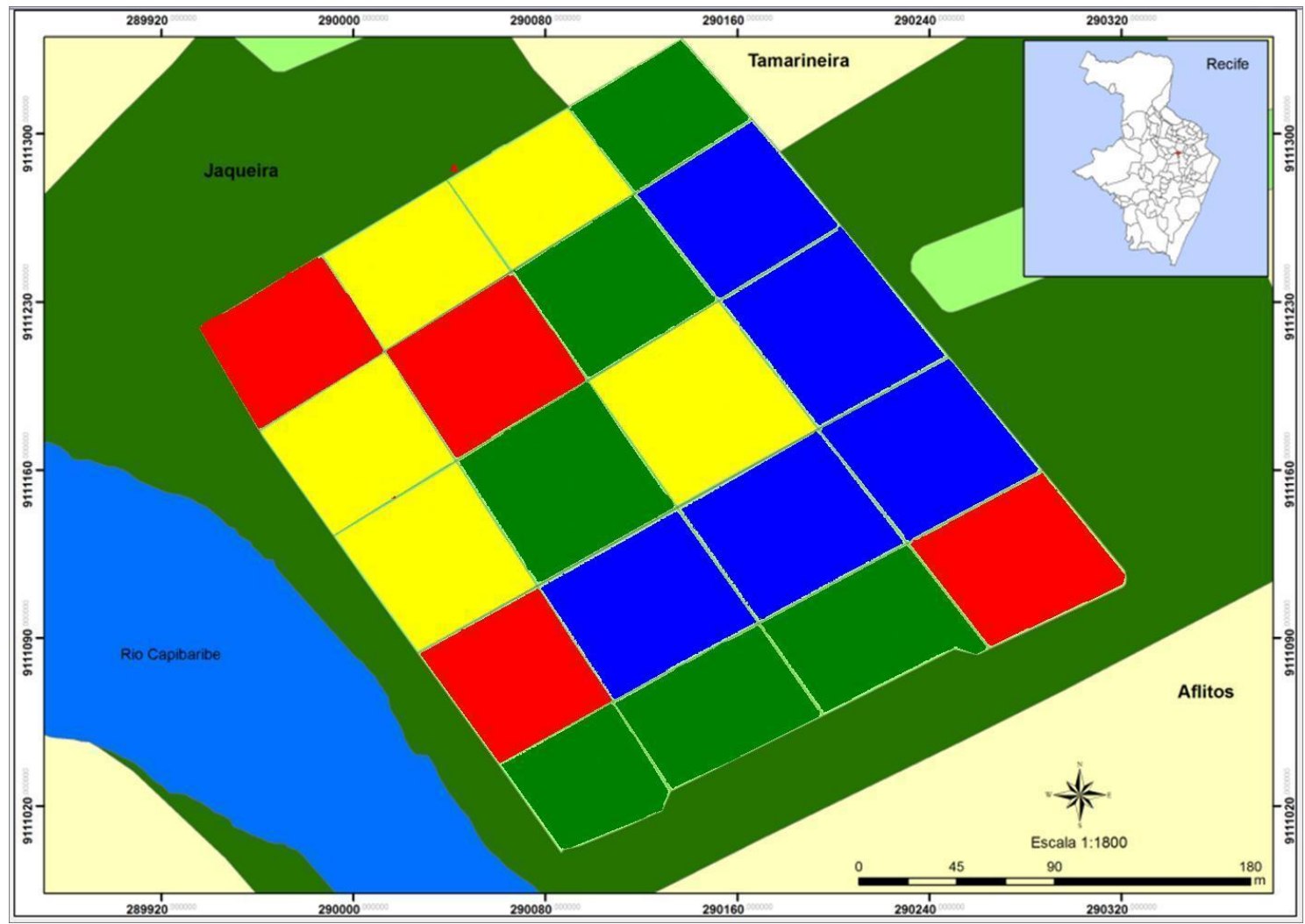

Fonte: IBGE, 2001. Licença ArcGIS 9.3 fornecida pelo SERGEO/UFPE.

Legenda: verde (muito bom); azul (bom); amarelo (médio); vermelho (baixo).

Os dados revelam ainda que os quadrantes entre os níveis 6 e 18 são aqueles considerados como de mais baixa qualidade do ar, equivalendo a $20 \%$ da área analisada. Os demais $(25 \%)$ são quadrantes classificados como de valores intermediários de IPA, condicionados por áreas de construção civil e com número reduzido de forófitos, bem como pelo intenso fluxo de veículos nas principais vias que circundam o parque.

Em estudo realizado por Cáceres (1996) no Campus da Universidade Federal de Pernambuco, com metodologia semelhante do uso de quadrantes, foi observada a influência do tráfego automotivo na diversidade e biomassa liquênica. Silva et al. (2009) realizaram uma atualização dos dados obtidos por Cáceres (1996) observando que decorridos 13 anos do estudo, os valores de IPA indicaram um aumento da poluição 
atmosférica. Dos 153 quadrantes analisados, em apenas 56\% havia liquens e destes, $50 \%$ estavam em níveis de poluição intermediários. Foi comprovado que as áreas de maior degradação eram aquelas cortadas por estacionamentos e tráfego de veículos, construções, e em quadrantes que possuíam poucos forófitos, ou por sua ausência. Esses fatores são determinantes para a diminuição da diversidade liquênica, posto que a arborização no meio urbano condiciona importante dispositivo capaz de remover material particulado emitido pelos veículos.

Com isso, é possível considerar o IPA método adequado para detectar e estimar a poluição atmosférica, visto que é técnica que independe das condições climáticas da área em estudo.Calvelo e Liberatore (2004) utilizaram esta técnica para avaliação da poluição atmosférica na cidade de Bariloche (Patagônia, Argentina), demonstrando que o método é uma forma confiável e relativamente barata de avaliar a poluição do ar em cidades. Os autores correlacionaram dados de IPA obtidos com as análises de danos nas células de algas dos liquens Flavoparmeliasoredians(Nyl.) Hale e Teloschisteschrysophthalmus(L.) Th. Fr. e verificaram que os valores de IPA tiveram aumento progressivo em função do afastamento do centro para as áreas periurbanas. Estes dados estavam em concordância com os obtidos em relação aos danos causados nas células de algas, que demonstraram uma degradação dos liquens nas zonas periurbanas da cidade, sendo isso atribuído possivelmente à poluição resultante do tráfego de veículos.

A sensibilidade de algumas espécies de liquens pode ser utilizada para monitorar a saúde de ambientes onde poluentes atmosféricos estão presentes (ROSENTRETER e ELDRIDGE, 2002; WILL-WOLF, 2002;NASH, 2008). Desta forma, os liquens podem ser usados para monitorar possíveis problemas de saúde humana (CISLAGHI e NIMIS 1997; CUNHA et al., 2007; NASH, 2008).

Diante de poluentes atmosféricos o organismo humano responde de forma diferenciada com irritação na garganta, ardência nos olhos, espirros, tosse, ou através de situações mais graves como o fechamento da glote, apneia, respiração rápida e superficial e constrição das vias aéreas inferiores. A exposição prolongada a certos poluentes possibilitam respostas crônicas do organismo que levam à caracterização de doenças ocupacionais (CUNHA, 2005). 
O uso de liquens como biomonitores da qualidade do ar tem se mostrado bastante eficiente. Silva (2002) analisou a concentração de metais pesados em área urbana no município de Jaboatão dos Guararapes, Pernambuco, a partir de transplantesdo líquen Cladoniaverticillaris(Raddi) Fr. comprovando alterações fisiológicasdevido às altas concentrações de $\mathrm{Ca}$, Fe e $\mathrm{Zn}$, com maior agressividade nas amostras que possuíam influência de tráfego de automotores.

Cunha et al. (2007) transplantarama mesma espéciepara uma indústria de extração e beneficiamento de calcário em Vertente do Lério, Agreste pernambucano, ratificando alterações na estrutura do líquen resultantes do impacto das partículas atmosféricas resultantes das explosões nas minas e dacalcinação dessa rocha, que liberava altos teores de $\mathrm{SO}_{2}$ para o ambiente, sendo este comportamento mais evidente nos transplantes que estavam próximos às indústrias e minas, ou em localidadesfavoráveis à direção predominante dos ventos.

A avaliação dos teores de clorofila está diretamente relacionada com os percentuais de células mortas. Sendo o líquen uma associação de alga e fungo, a morte de células verdes originárias das algas apresenta uma correlação com a queda na produção de clorofila, taxa de pigmentos fotossintetizantes e, consequentemente, morte do líquen (MAZZITELLIet al., 2006). Então, a direção predominante da micotaliquenizada nas áreas de estudo é, via de regra, a favor das correntes atmosféricas, forma pela qual a umidade e os nutrientes são carreados e absorvidos. Nesse contexto, Cáceres (1996) em biomonitoramento passivo da micotaliquênica existente no Campus da UFPE, ratificou sua predominância nos quadrantes Sul e Sudeste, onde na maior parte do ano predominam os alísios de Sudeste, que trazem a umidade marítima para a costa. Tais dados são corroborados por Seaward (1977), que ainda sugere serem os liquens indicadores de umidade e direção dos ventos.

A determinação dos teores de pigmentos fotossintéticos é um parâmetro útil, pois o contaminante ao entrar na célula degrada a clorofila, produto básico da fotossíntese, transformando-a em feofitina, ou destroi os cloroplastos, sítio de sua ocorrência. De uma forma ou de outra, o funcionamento básico do líquen é prejudicado por ser a fotossíntese o processo inicial de sua sobrevivência (MOTA-FILHOet al., 2003). Neste trabalho, as taxas de clorofila detectadas emP. Praesorediosum 
demonstraram que nos $55 \%$ dos quadrantes onde a espécie ocorria, um total de $73 \%$ possuía teores de clorofila superiores aos de feofitina(Tabela 2).

Tabela 2. Quantificação dos pigmentos fotossintetizantes de Parmotrema praesorediosiumem diferentes quadrantes delimitados no parque da Jaqueira.

\begin{tabular}{|c|c|c|c|c|c|c|}
\hline Quadrantes & $\begin{array}{c}\text { Clorofila } \\
\text { a }\end{array}$ & $\begin{array}{c}\text { Clorofil } \\
\text { a b }\end{array}$ & $\begin{array}{c}\text { Feofitin } \\
\text { a a }\end{array}$ & $\begin{array}{c}\text { Feofitin } \\
\text { a b }\end{array}$ & $\begin{array}{l}\text { Clorofil } \\
\text { a Total }\end{array}$ & $\begin{array}{c}\text { FeofitinaTota } \\
l\end{array}$ \\
\hline 1 & 7,34 & 3,10 & 8,18 & 1,92 & 5,22 & 5,05 \\
\hline 2 & $*$ & $*$ & $*$ & $*$ & $*$ & $*$ \\
\hline 3 & 10,72 & 4,56 & 12,93 & 1,18 & 7,64 & 7,06 \\
\hline 4 & 12,27 & 4,16 & 16,77 & $-2,58$ & 8,21 & 7,10 \\
\hline 5 & $*$ & $*$ & $*$ & * & $*$ & $*$ \\
\hline 6 & * & * & * & * & * & * \\
\hline 7 & 5,37 & 2,32 & 5,24 & 2,68 & 3,84 & 3,96 \\
\hline 8 & 0,94 & 0,58 & 0,50 & 1,28 & 0,76 & 0,89 \\
\hline 9 & 6,88 & 2,74 & 9,10 & $-0,76$ & 4,81 & 4,17 \\
\hline 10 & 14,18 & 5,25 & 19,63 & $-2,95$ & 9,71 & 8,34 \\
\hline 11 & $*$ & * & * & $*$ & * & * \\
\hline 12 & $*$ & * & $*$ & $*$ & $*$ & * \\
\hline 13 & 2,99 & 1,26 & 3,18 & 1,08 & 2,12 & 2,13 \\
\hline 14 & $*$ & * & $*$ & $*$ & $*$ & $*$ \\
\hline 15 & 13,03 & 5,29 & 17,63 & $-2,37$ & 9,16 & 7,63 \\
\hline 16 & $*$ & $*$ & $*$ & $*$ & $*$ & $*$ \\
\hline 17 & 7,33 & 2,72 & 10,35 & $-2,12$ & 5,02 & 4,12 \\
\hline 18 & * & $*$ & $*$ & $*$ & $*$ & $*$ \\
\hline 19 & $*$ & $*$ & $*$ & $*$ & $*$ & $*$ \\
\hline 20 & 21,99 & 8,31 & 32,39 & $-9,15$ & 15,15 & 11,62 \\
\hline
\end{tabular}

Legenda: * quadrantes sem ocorrência da espécie.

Os resultados demonstram que apesar de haver teores de clorofila superiores aos de feofitina, alguns quadrantes possuíam taxas muito equiparadas devido, 
provavelmente a um processo de degradação da clorofila em feofitina.Richardson e Pucckett (1973) afirmaram que as feofitinas são resultantes de um processo de degradação das clorofilas, a partir da perda do íon de $\mathrm{Mg}^{2+}$, que está presente no centro de sua estrutura química. Essa perda pode ser ocasionada pela acidificação do meio, degradando a clorofila em feofitina. No caso dos poluentes, estes entram de forma acidificada na célula do líquen,degradando as clorofilas. Dessa forma, influi na fotossíntese, provocando alterações no seu metabolismo. Cáceres (1996) observou que o líquen passa por um processo de degradação de sua estrutura e funcionamento, a partir da ação dos poluentes. Em relação aos pigmentos, inicialmente há uma lenta degradação de clorofila e aumento da feofitina, ficando seus valores próximos. Em seguida, os teores vão se invertendo até uma predominância da feofitina. Nessa situação, a poluição está em estágio avançado e os liquens em fase de desaparecimento. Por isso, o uso desta técnica consiste em uma detecção precoce dos efeitos da poluição, onde os danos visíveis à biota ainda não são evidentes (MOTA-FILHOet al., 2003). Dessa forma, o método é amplamente utilizado em outras parte do mundo, independentemente das características físico-climáticas, ou especies de liquens dominantes na área. A exemplo disso, refere-se o trabalho de Carreras et al. (1998)que utilizaram Usneasp no biomonitoramento de cinco zonas da cidade de Córdoba, Argentina, em áreas industriais e de intenso tráfego. Os autores observaram que houve uma degradação da clorofila a devido, principalmente, à presença de enxofre expelido pelas indústrias.

Dentre os quadrantes analisados no parque da Jaqueira, para a verificação das taxas de clorofila e feofitina constatou-se que os quadrantes 10,15 e 20 foram os que obtiveram os maiores valores de clorofila devido ao fato de estarem mais afastados das vias de tráfego, bem como seus quadrantes terem um número elevado de forófitos, já que as árvores funcionam como uma barreiras aos poluentes atmosféricos, acarretando num ambiente a eles menos exposto, proporcionando um microclima local.

Araújo et al. (2009) em análise de pigmentos fotossitetizantes de $P$. praesorediosium no Campus da UFPE, verificaram que em apenas $28 \%$ dos 86 quadrantes analisados, foi encontrada a espécie. Foram considerados fatores determinantes para essa baixa ocorrênciao número crescente deedificações no Campus, a existência da $\mathrm{BR}$ - 101Sul muito próxima àsua entrada e, a direção predominante dos 
ventos no sentido $\mathrm{S} / \mathrm{SE}$, perpendicular a essa rodovia que propicia a disseminação dos gases expelidos pelos veículos automotores.

Tanto em biomonitoramento com espécies nativas na área de estudo (passivo), como com material transplantado de áreas sem fontes poluidoras, as emissões industriais e tráfego de veículos são os maiores produtores de danos à biota. Assim, espécies como Leptogiumsp e $P$. praesorediosum usadas em biomonitoramento passivo, ou $C$. verticillaris espécie preferida para experimentos de transplantes, demonstram teores crescentes de feofitinas à medida que são detectados mais altos níveis de contaminantes (CÁCERES, 1996; MOTA-FILHOet al., 2003; 2007),demonstrando a sensibilidade do líquen aos contaminantes atmosféricos, principalmente $\mathrm{SO}_{2}$ e $\mathrm{NO}_{2}$.

Portanto, a validação de uma espécie é, sobretudo, importante para a confiabilidade dos resultados. C. verticillaris foi avaliada em conjunto com monitores mecânicos na cidade do Recife, Jaboatão dos Guararapes, cujos resultados ratificaram sua eficácia como biomonitor (CÁCERES, 1996; SILVA, 2002). A partir daí outros estudos se seguiram como o de Freitas (2006) que analisou os teores de clorofila de transplantadas em áreas do Complexo Industrial Portuário de Suape, Pernambuco. O autor verificou que em todos os pontos de coleta foram observadas taxas de feofitina superiores as de clorofila, sobretudo onde os ventos transportavam altas cargas de fluoretos emitidos por uma indústria de cerâmica. Nos pontos com unidades fabris que usavam tecnologias limpas, os biomonitores responderam de forma satisfatória. Dessa forma, foi possível constatar que os liquens respondem com alterações morfológicas e fisiológicas às distintas doses de poluentes a que estes são expostos, permitindo atribuir diferentes níveis de contaminação atmosférica.

\section{CONCLUSÃO}

A correlação dos dados do IPA obtidos com liquens ocorrentes no parque da Jaqueira com os teores de pigmentos de $P$. praesorediosium, demonstra que o parqueé uma área parcialmente poluída, visto que $45 \%$ de sua área não possuem a espécie analisada quimicamente, com predomínio de espécies crostosas, que são mais resistentes à poluição do que as foliosas ou fruticosas. Na área onde há ocorrência de $P$. praesorediosium os dados de IPA indicaram o ar como de boa a alta qualidade e, a cobertura arbórea demonstra ser um fator de proteção à micota liquenizada. 
Este fato pode ser condicionado pelo tráfego de veículos, principalmente pela Avenida Rui Barbosa, que é uma das vias mais movimentadas da cidade do Recife, sendo estalocalizada na entrada do parque, o qual também possui a influência do rio Capibaribe que corta a cidade, cuja umidade transportada à cobertura vegetal pode agregar poluentes particulados ou não, absorvidos pela micota liquenizada.

Destaca-se a necessidade de uma reestrutuação do Parque a partir da implantação de espécies arbóreas na área próxima à Avenida Rui Barbosa. Tal gestão poderia auxiliar na formação de uma barreia ao poluentes, na amenização climática e consequente aumento na diversidade de espécies.

\section{AGRADECIMENTOS}

À Coordenação de Aperfeiçoamento de Pessoal de Nível Superior (CAPES) pela concessão das Bolsasde Mestrado de AKOS e IMCP, e ao CNPq pela Bolsade Produtividade em Pesquisa (PQ) de ECP.

\section{REFERÊNCIAS}

ARAÚJO, E. L.; SILVA, A. K. O. ; SILVA, A. K. B. B.; PEREIRA, I. M. C.; VASCONCELOS, R. D.; CAVALCANTI, S.A.; VASCONCELOS, T. L.; PEREIRA, E.G.; SILVA, N. H. Quantificação dos teores de clorofila e feofitina de Parmotremapraesorediosium no Campus da Universidade Federal de Pernambuco Brasil. In: IX Encontro do Grupo Latinoamericano de Liquenólogos, 2009, Corrientes. IX Encuentrodel Grupo Latinoamericano de Liquenólogos, 2009. v. 01. p. 23-23.

AYOADE, J.O. Introdução a climatologia para os trópicos. Rio de Janeiro: Bertrand Brasil, 1998. 332 p.

CÁCERES, M. E. S. Biomonitoramento ativo e passivo da qualidade do ar atmosférico na cidade do Recife. Monografia de Bacharelado em Ciências Biológicas. Universidade Federal de Pernambuco, Recife, 1996. 77 p.

CALVELO, S.; LIBERATORE, S. Applicability of in situor transplanted lichen for assessment of atmospheric pollution in Patagonia, Argentina. In: Journal of Atmospheric Chemistry, 49. 199 - 210, 2004.

CARRERAS, H. A.; GUDINO, G. L.; PIGNATA, M. L. Comparative biomonitoring of atmospheric quality in five zones of Cordoba City (Argentina) employing the transplanted lichen Usneasp.In: Environmental Pollution, 103, 317-325, 1998. 
CISLAGHI, C.; NIMIS, P. L. Lichens, air pollution and lung cancer. In: Nature, 387,463-464. 1997.

CUNHA, M. H. A.; SILVA, J. M.; MOTA FILHO, F. O.; SILVA, N. H.; PEREIRA, E. C. G. Cladoniaverticillaris(RADDI) Fr., para diagnostico da solubilidade do ar decorrente da extração e beneficiamento do calcário em Vertente do Lério, Pernambuco (Brasil).In: Revista Caminhos de Geografia Uberlândia v. 8, n. 22 set/2007. p. 49 65.

CUNHA, M. H. A. Biomonitoramento da qualidade do ar em Vertente do Lério PE, e sua correlação com ocorrência de doenças pulmonares. Dissertação de Mestrado em Gestão e Políticas Ambientais. Universidade Federal de Pernambuco, Recife, 2005. 102 p.

DE SLOOVER, J.; LEBLANC, F. Mapping of atmosferic pollution on the basis of lichen sensitivity. apud Leblanc, F; Rao, D.N. Effects of pollutants on lichens and bryophytes. In: Mudd, J.B.; Kozlowski, T.T. Response of plants to air pollution. London. 11:237-272. 1968.

FREITAS, F. M. R. Uso de Cladoniaverticillaris (Raddi) Fr. (líquen) como biomonitor da qualidade do ar no Complexo Industrial Portuário de SUAPE - PE. Dissertação de Mestrado em Biologia Vegetal. Universidade Federal de Pernambuco, Recife, 2006. 58 p.

FUGA, A.; SAIKI, M.; MARCELlI, M. P.; SALDINA, P. H. N. Atmospheric pollutants monitoring by analysis of epiphytic lichens. In: Environmental Pollution 151, 334-340, 2008.

HAWKSWORTH, D. L. Litmus tests for ecosystem health: the potential of bioindicator in the monitoring of biodiversity. In: SWAMINATHAN, M. S.; JANA, S. Biodiversity. Implications for global food security. Madras: Macmillan India 17, 184-204, 1992.

HILL, R. Chlorophyll. In: FLORKIN, M.; STOTZ, H. Comprehensive Biochemistry. vol. 9, pp. 73, 1963.

KLUMPP, A.; ANSEL, W.; KLUMPP, G.; FOMIN, A. Um novo conceito de monitoramento e comunicação ambiental: a rede européia para a avaliação da qualidade do ar usando plantas bioindicadoras (EuroBionet). In: Revista Brasileira de Botânica, São Paulo, V. 24, n. 4, p. 511-518, dez. 2011.

LEBLANC, F; DE SLOOVER, J. Relation between industrialization and the distribution and growth of epiphytic lichens and mosses in Montreal. In:Canadian Journal of Botany, 48, 1485-1496. 1970.

LE BLANC, F.; RAO, D. N. Effects of pollutants on lichens and bryophytes. In: MUDD, J. B.; KOZLOWSKI, T. T. Responses of plants to air pollution. London, Academic, Press, Inc. 11, 237-272, 1975. 
MARTINELLI, M. Cartografia temática: caderno de mapas. São Paulo: EDUSP, 2003. $160 \mathrm{p}$.

MAZZITELli, S. M. A. M.; MOTA FILHO, F. O.; PEREIRA, E. C. G.; FIGUEIRA, R. Utilização de liquens no biomonitoramento da qualidade do ar. In: Xavier-Filho, L.; Legaz, M. E.; Vicente, C.; Legaz, M. E.; Pereira, E. C. eds. Biologia de liquens. 1ed. Editora Âmbito Cultural, Rio de Janeiro, 2006. Pp. 99 - 143.

MOTA FILHO, F. O.; PEREIRA, E. C. G.; SILVA, R. A.; XAVIER FILHO, Lauro. Liquens: Bioindicadores ou biomonitores? (2003) In: Portal Biomonitor. Disponível em:

$<$ http://biomonitor.ist.utl.pt/biomonitor/index2.php?option=com_content\&do_pdf=1\&id =9. Acesso em: 05 de maio de 2010 .

MOTA FILHO, F. O.; PEREIRA, E. C. G.; LIMA, E. S.; SILVA, N.H.; FIGUEIREDO, R. C. B. Influência de poluentes atmosféricos em Belo Jardim (PE) utilizando Cladoniaverticillaris(líquen) como biomonitor. In: Química Nova, Vol. 30, No. 5, 1072-1076, 2007.

NASH III, T. H. Lichen sensitivity to air pollution. In: Lichen Biology. 2 ed. Cambridge, São Paulo, 2008. 486 p.

NIEBOER, E.; AHMED, H. M.; PUCKETT, K. J.; RICHARDSON, D. H. S. The heavy metalcontent of lichens in relation to distance from a nickel smelter in Sudbury, Ontario.Lichenologist, 1972, 5: 292 -304p.

NOWAK, D. J.; CIVEROLO, K. L.; RAO, S. T.; SISTLA, G.;

LULEY, C. J.; CRANE, D. E. A modeling study of the impact of urban trees on ozone.

PILEGAARD, K. Airborne metals and $\mathrm{SO}_{2}$ monitored by epiphytic lichens in an industrial area. In: Environ. Pollut 17, 81-91, 1978.

PREFEITURA DO RECIFE, (Município). Secretaria de Meio Ambiente da Cidade do Recife. Recife, PE, 2011.

RICHARDSON, D. H. S.; PUCKETT, K. J. Sulphur dioxide and photosynthesis in lichens. In: FERRY, B. W.; BADDELEY, M. S.; HAWKSWORTH, D. L. Air pollution and lichens. London, The Atholone Press, 283-298, 1973.

RIBEIRO, S. M. A.; GALHARDO, I. N.; PAIVA, R. S.; PEREIRA, E. C. G.; MOTAFILHO, F. O. ; SILVA, N. H. Determinação do índice de pureza atmosférica no Museu Paraense Emílio Goeldi (MPEG), através da analise da micotaliquenizada. In: XLVIII CongressoNacional de Botânica. v. 1.p. 153-154.1997.

ROSENTRETER, R.; ELDRIDGE, D. J. Monitoring biodiversity and ecosystem function: grasslands, deserts, and steppe. In: Monitoring with Lichens - Monitoring Lichens. Nato Science Series IV: Earth and Environmental Sciences, ed. P. L. Nimis, C. Scheidegger and P. A. Wolseley, pp. 223-237. Dordrecht: Kluwer Academic. 2002. 
SEAWARD, M. R. D. Lichen Ecology. London, Academic Press, Inc. 1977. 550 p.

SEAWARD, M. R. D. Lichen and sulphur dioxide air pollution: field studies. In: Eviron. Rev. 1, 73-91, 1993.

SILVA, A. K. O.; SILVA, A. K. B. B.; ARAUJO, E. L.; PEREIRA, I. M. C.; VASCONCELOS, T. L.; PEREIRA, E. C. G.; SILVA, N. H. Avaliação do Índice de Pureza Atmosférica no Campus da Universidade Federal de Pernambuco - Brasil. In: IX Encontro do Grupo Latinoamericano de Liquenólogos, 2009, Corrientes. IX Encuentrodel Grupo Latinoamericano de Liquenólogos, 2009. v. 01. p. 58-58.

SILVA, R. A. Cladoniaverticillaris(líquen) como biomonitor padrão da qualidade do ar no Distrito de Jaboatão - PE. Dissertação de Mestrado em Gestão e Políticas Ambientais. Universidade Federal de Pernambuco, Recife, 2002. 159 p.

VERNON, L. P. Spectrophotometric determination of chlorophyll and phaeophytins in plants extracts. In: Anal. Biochem. 32, 11-42, 1960.

WILL-WOLF, S. Monitoring regional status and trends in forest health with lichen communities: the United States Forest Service approach. In:Monitoring with Lichens - Monitoring Lichens, ed. P. L. Nimis, C. Scheidegger and P. A. Wolseley, pp. 353-357. Nato Science Series IV: Earth and Environmental Sciences.

Dordrecht: Kluwer Academic Publishers. 2002.

YAAKOV, Y.; POTCHTER, O.; BITAN. A. The influence of a small urban park on climatical parameters: a case study of Gan-Meir, Tel-Aviv. In: Climate and environmental change - Pre-Regional Conference Meeting of the Commission on Climatology, 1998, Anais. Lisboa: International Geographical Union CommissiononClimatology, 1998. p. 03-04.

Artigo recebido para publicação em jan/14

Artigo aceito para publicação em março/14. 